\title{
On the Sphere Problem
}

\section{Fernando Chamizo and Henryk Iwaniec}

\section{Introduction and Statement of Main Results.}

One of the oldest problems in analytic number theory consists of counting points with integer coordinates in the $d$-dimensional ball. It is very easy to find a main term for the counting function, but the size of the error term is difficult to estimate. Namely the problem is to prove the approximate formula

$$
\#\left\{\mathbf{x} \in \mathbb{Z}^{d}:\|\mathbf{x}\| \leq R\right\}=\frac{\pi^{d / 2}}{\Gamma\left(\frac{d+2}{2}\right)} R^{d}+O\left(R^{\theta}\right),
$$

for any $R \geq 1$ with $\theta$ as small as possible. Let $\theta_{d}$ be the least number such that the above approximation is true with any $\theta>\theta_{d}$.

In dimension $d=1, \theta_{1}=0$ follows trivially. The problem is also settled when $d \geq 4$. In this case, starting from a classical formula for the number of representations as sum of four squares and using elementary arguments, it can be proved $\theta_{d}=d-2$ (see for instance [Fr]).

The evaluation of $\theta_{d}$ in the remaining cases $d=2, d=3$ is an outstanding problem in number theory and intractable by the methods of nowadays. The conjectures (supported by some mean results) are $\theta_{2}=1 / 2, \theta_{3}=1$.

The two dimensional problem is called "the circle problem" and it has a long history coming back to Gauss, who proved $\theta_{2} \leq 1$. In this century several authors gave some improvements of this result, very often creating new methods in the theory of exponential sums; the best result so far is $\theta_{2} \leq 46 / 73$ due to Huxley [Hu]. 
The three dimensional case, the so called "sphere problem", is also closely related with the work of Gauss about the average of the class number for negative discriminants (see Art. 302 of [Ga]). The literature about the sphere problem is not so wide as in the two dimensional case, although it seems more interesting because it has profound relations with others topics in number theory: class number, $L$-functions, etc. The best result until now was $\theta_{3} \leq 4 / 3$ due to Chen [Ch] and Vinogradov [Vi]. The purpose of this paper is to improve this bound $\left(\theta_{3} \leq 29 / 22\right.$, see Theorem 1.1).

Before stating our main theorem we shall introduce some notation.

First of all we define $r_{3}(n)$, for a positive integer $n$, to be the number of representations as sum of three squares

$$
r_{3}(n)=\#\left\{\left(n_{1}, n_{2}, n_{3}\right) \in \mathbb{Z}^{3}: n_{1}^{2}+n_{2}^{2}+n_{3}^{2}=n\right\}
$$

and $R_{3}(n)$ to be the number of primitive representations

$R_{3}(n)=\#\left\{\left(n_{1}, n_{2}, n_{3}\right) \in \mathbb{Z}^{3}: \operatorname{gcd}\left(n_{1}, n_{2}, n_{3}\right)=1, n_{1}^{2}+n_{2}^{2}+n_{3}^{2}=n\right\}$.

These two functions are related by the formula

$$
r_{3}(n)=\sum_{d^{2} \mid n} R_{3}\left(\frac{n}{d^{2}}\right) .
$$

There are also the following relations with other arithmetic quantities (see $[\mathrm{Gr}])$ :

$$
R_{3}(n)=c_{n} h(-4 n)=\frac{1}{\pi} c_{n} \sqrt{n} L\left(1, \chi_{n}\right), \quad n>1,
$$

where $h(-4 n)$ is the class number for the negative discriminant $-4 n$, $L\left(s, \chi_{n}\right)$ is the $L$-function associated with the character

$$
\chi_{n}(m)=(-4 n / m)
$$

and

$$
c_{n}=\left\{\begin{aligned}
0, & \text { if } n \equiv 0,4,7(\bmod 8) \\
16, & \text { if } n \equiv 3(\bmod 8), \\
24, & \text { if } n \equiv 1,2,5,6(\bmod 8) .
\end{aligned}\right.
$$

In order to write some formulas in a more symmetric way, we normalize $r_{3}(n)$ by

$$
l(n)=r_{3}(n) n^{-1 / 2}
$$


The previous formulas show that $l(n)$ is very similar to $L\left(1, \chi_{n}\right)$. In fact our method is based in that we can consider a sum of $l(n)$ as a lattice point problem or as a sum of character sums. This duality allows us to employ, in different ranges, Poisson's summation or Burgess' inequality $[\mathrm{Bu}]$.

Let $S(R)$ be the number of lattice points in the sphere of radius $R$ and $S(R, H)$ be the number of lattice points between the spheres of radius $R$ and $R+H$, i.e.

$$
S(R)=\sum_{n \leq R^{2}} r_{3}(n) \quad \text { and } \quad S(R, H)=\sum_{R^{2}<n \leq(R+H)^{2}} r_{3}(n) .
$$

Our main results are the following:

Theorem 1.1. For any $R \geq 1 \geq H>0$ and $\varepsilon>0$ we have

$$
S(R)=\frac{4 \pi}{3} R^{3}+O\left(R^{29 / 22+\varepsilon}\right)
$$

and

$$
S(R, H)=4 \pi H R^{2}+O\left(\left(H^{7 / 8} R^{15 / 8}+H^{2 / 3} R^{83 / 48}+R\right) R^{\varepsilon}\right)
$$

REMARK. One should be able to establish an asymptotic formula for the mean value of $h(-n)$ with an error term as good as in Theorem 1.1. We intend to deal with this problem in another occasion.

\section{A summation formula for $l(n)$.}

In this section we shall establish

Lemma 2.1. If $f \in C_{0}^{3}([0, \infty))$ with $f^{\prime \prime}(0)=0$ then

$$
\sum_{n=1}^{\infty} l(n) f(\sqrt{n})=4 \pi \int_{0}^{\infty} r f(r) d r-f^{\prime}(0)+\sum_{n=1}^{\infty} l(n) \widetilde{f}(\sqrt{n}),
$$

where $\tilde{f}$ is the sine Fourier transform

$$
\tilde{f}(\xi)=2 \int_{0}^{\infty} f(x) \sin (2 \pi x \xi) d x .
$$


Proof. By our hypothesis on $f$, the function

$$
g(x, y, z)=\frac{f\left(\sqrt{x^{2}+y^{2}+z^{2}}\right)}{\sqrt{x^{2}+y^{2}+z^{2}}}
$$

can be extended to a $C_{0}^{2}$ function in $\mathbb{R}^{3}$ and then by the classical Poisson summation formula we get

$$
f^{\prime}(0)+\sum_{n=1}^{\infty} l(n) f(\sqrt{n})=\sum_{n_{1}, n_{2}, n_{3}} \widehat{g}\left(n_{1}, n_{2}, n_{3}\right) .
$$

The Fourier transform of a radial function is radial, therefore we can suppose that the point $\left(n_{1}, n_{2}, n_{3}\right)$ is on the $z$-axis, in which case by a change to spherical coordinates it follows easily that

$$
\widehat{g}\left(n_{1}, n_{2}, n_{3}\right)=\frac{\widetilde{f}\left(\sqrt{n_{1}^{2}+n_{2}^{2}+n_{3}^{2}}\right)}{\sqrt{n_{1}^{2}+n_{2}^{2}+n_{3}^{2}}}, \quad \text { if } n_{1}^{2}+n_{2}^{2}+n_{3}^{2} \neq 0
$$

and

$$
\widehat{g}(0,0,0)=4 \pi \int_{0}^{\infty} r f(r) d r,
$$

hence the proof is complete.

\section{Exponential sums over lattice points in spheres.}

If we had chosen $f(x)=x$ for $0<x<R$ in Lemma 2.1 (actually one has to make some smoothing) then we could infer that

$$
S(R)=\frac{4 \pi}{3} R^{3}+O\left(R^{3 / 2+\varepsilon}\right),
$$

or equivalently $\theta_{3} \leq 3 / 2$. This result was first proved by Landau [La], other better results were established by Walfisz [Wa1], Fomenko [Fo], Chen [Ch] and Vinogradov in several papers culminating in [Vi]. As we quoted in the introduction, the best exponent in the error term until now was $4 / 3+\varepsilon$ due to Chen and Vinogradov (in fact Vinogradov replaced $R^{\varepsilon}$ by a logarithmic factor). These results require non-trivial estimates for exponential sums of the type

$$
V_{N}(R)=\sum_{n \asymp N} r_{3}(n) e(R \sqrt{n})
$$


where here and thereafter we write $n \asymp N$ to say that $c_{1} N<n<c_{2} N$ with some unspecified constants $c_{1}, c_{2}$, not necessarily the same ones in each ocurrence.

We prove in this section (by arguments similar to those in Chen [Ch] and Vinogradov [Vi])

Lemma 3.1. For $R>1$ we have

$$
\begin{aligned}
V_{N}(R) \ll N^{5 / 4+\varepsilon}+N^{\varepsilon} \min \{ & R^{3 / 8} N^{15 / 16}+R^{1 / 8} N^{17 / 16}, \\
& \left.R^{7 / 24} N^{49 / 48}+R^{5 / 24} N^{53 / 48}\right\} .
\end{aligned}
$$

Proof. We shall deduce that

$$
\begin{aligned}
V_{N}(R) & \ll\left|\sum_{a, b, c} e\left(R \sqrt{a^{2}+b^{2}+c^{2}}\right)\right| \\
& \ll N^{\varepsilon} \sum_{n \asymp N}\left|\sum_{c \ll \sqrt{N}} e(\theta c) e\left(R \sqrt{n+c^{2}}\right)\right|
\end{aligned}
$$

for some $\theta \in \mathbb{R}$. To prove the above we select the smallest variable, say $c$, and apply [Gr-Ko, Lemma 7.3] for the sum over $c$ in order to remove the involved summation conditions. From the variables $a, b$ we create a new variable $n=a^{2}+b^{2}$. Splitting the range of the inner sum into segments of lenght $N^{1 / 2-\varepsilon}$ by Cauchy's inequality,

$$
V_{N}^{2}(R) \ll N^{1+\varepsilon} \sum_{c_{1}, c_{2}}\left|\sum_{n \asymp N} e\left(R\left(\sqrt{n+c_{1}^{2}}-\sqrt{n+c_{2}^{2}}\right)\right)\right|
$$

where $c_{1}, c_{2}$ are restricted by $c_{1}, c_{2}<N^{1 / 2}$ and $\left|c_{1}-c_{2}\right|<N^{1 / 2-\varepsilon}$. Hence for a suitable $D<N^{1-\varepsilon}$ we get

$$
V_{N}^{2}(R) \ll N^{5 / 2+\varepsilon}+N^{1+\varepsilon} \sum_{y \asymp D}\left|\sum_{x \asymp N} e(f(x, y))\right|
$$

where

$$
f(x, y)=R(\sqrt{x}-\sqrt{x+y}) .
$$

If $D \ll N^{3 / 2} R^{-1}$ the innermost sum is $\ll N^{3 / 2} R^{-1} D^{-1}$ by [Gr-Ko, Theorem 2.1] of so it contributes $\ll N^{5 / 2+\varepsilon}$. 
If $D \gg N^{3 / 2} R^{-1}$ we apply the $B$-process of the one-dimensional van der Corput's method (Poisson sumation and stationary phase), more precisely [Gr-Ko, Lemma 3.6] of with $F=R D N^{-1 / 2}$ getting

$$
\begin{aligned}
V_{N}^{2}(R) \ll & N^{5 / 2+\varepsilon}+R^{-1 / 2} D^{1 / 2} N^{9 / 4+\varepsilon} \\
& +R^{-1 / 2} D^{-1 / 2} N^{9 / 4+\varepsilon} \sum_{y \asymp D}\left|\sum_{x \asymp U} e(g(x, y))\right|
\end{aligned}
$$

where $U=R D N^{-3 / 2}$,

$$
g(x, y)=f(\alpha(x, y), y)-x \alpha(x, y)
$$

and $\alpha(x, y)$ is the implicit function defined by

$$
f_{x}(\alpha(x, y), y)=x .
$$

The middle term in (3.1) comes from the error term in [Gr-Ko, Lemma 3.6].

Next, by Cauchy's inequality and dividing into dyadic intervals, there exists $1 \leq T<U$ such that

$$
V_{N}^{4}(R) \ll N^{5+\varepsilon}+R^{-1} D N^{9 / 2+\varepsilon}+R^{-1} N^{9 / 2+\varepsilon} V_{U T D}
$$

where

$$
V_{U T D} \ll \sum_{x \asymp U} \sum_{z \asymp T}\left|\sum_{y \asymp D} e(G(x, y, z))\right|
$$

with

$$
G(x, y, z)=g(x+z, y)-g(x, y) .
$$

Now we apply two well known van der Corput's estimates (see [Gr-Ko, Theorem 2.2] and [Ti, Theorem 5.11] to obtain

$$
V_{U T D} \ll U T \min \left\{D \lambda_{2}{ }^{1 / 2}+\lambda_{2}{ }^{-1 / 2}, D \lambda_{3}{ }^{1 / 6}+D^{1 / 2} \lambda_{3}{ }^{-1 / 6}\right\}
$$

where $\lambda_{2} \asymp\left|G_{y y}\right|$ and $\lambda_{3} \asymp\left|G_{y y y}\right|$. Here, by the mean value theorem $\lambda_{2} \asymp T\left|g_{x y y}\right|$ and $\lambda_{3} \asymp T\left|g_{x y y y}\right|$. On the other hand, by (3.2) and (3.3) $g_{x}=-\alpha$, hence $\lambda_{2} \asymp T\left|\alpha_{y y}\right|$ and $\lambda_{3} \asymp T\left|\alpha_{y y y}\right|$.

It remains to estimate the partial derivatives $\alpha_{y y}, \alpha_{y y y}$. By the definition of $f$ and (3.3) we have $\alpha^{-1 / 2}-(\alpha+y)^{-1 / 2}=2 x R^{-1}$. Differenciating with respect to $y$ and eliminating $\alpha+y$, we get

$$
\frac{1}{\alpha_{y}}=\left(1+\frac{y}{\alpha}\right)^{3 / 2}-1 \quad \text { and } \quad \frac{\alpha_{y}}{\alpha_{y}+1}=(1-h)^{3}
$$


with

$$
h=\frac{2 x \alpha^{1 / 2}}{R} \asymp \frac{D}{N} \ll N^{-\varepsilon} .
$$

The first formula (recalling $y \asymp D, \alpha \asymp N$ ) implies $\alpha_{y} \asymp N D^{-1}$ and differentiating the second formula we obtain

$$
-\alpha_{y y}=6 x^{2} R^{-2} h^{-1}(1-h)^{2} \alpha_{y}\left(\alpha_{y}+1\right)^{2} .
$$

Using $\alpha_{y}=(1-h)^{3} /\left(1-(1-h)^{3}\right)$ we get

$$
-\alpha_{y y}=6 x^{2} R^{-2} h^{-1}(1-h)^{5}\left(1-(1-h)^{3}\right)^{-3} .
$$

Since $h \asymp D N^{-1}$ and $x \asymp U=R D N^{-3 / 2}$, this formula gives $\left|\alpha_{y y}\right| \asymp$ $N D^{-2}$. Differentiating again

$$
\alpha_{y y y}=\frac{h^{2}(2-h)}{3(1-h)^{6}}\left(3-3 h+h^{2}\right)^{2}\left(5 h^{2}-6 h+6\right) \alpha_{y y}^{2} \alpha_{y}
$$

whence $\alpha_{y y y} \asymp N D^{-3}$. From the above estimates for the partial derivatives we conclude that

$$
\lambda_{2} \asymp T N D^{-2} \quad \text { and } \quad \lambda_{3} \asymp T N D^{-3} .
$$

Substituting in (3.4) and (3.5) we have

$$
\begin{aligned}
& V_{N}^{4}(R) \ll N^{5+\varepsilon}+R^{-1} D N^{9 / 2+\varepsilon} \\
&+N^{\varepsilon} R^{-1} U \min \{ T^{3 / 2} N^{5}+T^{1 / 2} D N^{4}, \\
&\left.T^{7 / 6} D^{1 / 2} N^{14 / 3}+T^{5 / 6} D N^{13 / 3}\right\} .
\end{aligned}
$$

Finally, recalling that $U=R D N^{-3 / 2}, T<R D N^{-3 / 2}$ and $N^{3 / 2} R^{-1} \ll$ $D<N$ the lemma follows.

\section{A character sum estimate.}

The objective of this section is to proof the following character sum estimate

Lemma 4.1. For $1<K<N^{1 / 2}$ and $\alpha_{n}$, $\beta_{n}$ arbitrary complex numbers

$$
\begin{aligned}
& \sum_{N<n \leq N+K} \sum_{m \asymp M} \alpha_{n} \beta_{m}\left(\frac{n}{m}\right) \\
& \ll\|\alpha\|\|\beta\|\left(K^{3 / 8} M^{1 / 2}+K^{1 / 2} M^{1 / 4} N^{3 / 64}\right)(M N)^{\varepsilon} .
\end{aligned}
$$


As a corollary it can be obtained the following $L$-function estimate

Corollary 4.2. If $1<K<N^{1 / 2}$

$$
\sum_{\substack{N<n \leq N+K \\ n \equiv \nu(8)}} L\left(1, \chi_{n}\right)=\frac{3 \zeta(2)}{28 \zeta(3)} K+O\left(K^{7 / 8} N^{\varepsilon}+K^{2 / 3} N^{1 / 32+\varepsilon}\right) .
$$

Proof of Lemma 4.1. Dividing the range of summation into dyadic intervals, it is enough to estimate

$$
S_{K M}=\sum_{k \asymp K} \sum_{m \asymp M} \alpha_{N+k} \beta_{m}\left(\frac{N+k}{m}\right) .
$$

By Cauchy's inequality

$$
S_{K M}^{2} \ll\|\alpha\|^{2} \sum_{k \asymp K}\left|\sum_{m \asymp M} \beta_{m}\left(\frac{N+k}{m}\right)\right|^{2} .
$$

Again by Cauchy's inequality and interchanging the order of summation

$$
\begin{aligned}
S_{K M}^{4} & \ll\|\alpha\|^{4} K \sum_{k \asymp K}\left|\sum_{m \asymp M} \beta_{m}\left(\frac{N+k}{m}\right)\right|^{4} \\
& =\|\alpha\|^{4} K \sum_{\substack{m_{1}, m_{2} \\
m_{3}, m_{4}}} \beta_{m_{1}} \beta_{m_{2}} \bar{\beta}_{m_{3}} \bar{\beta}_{m_{4}} \sum_{k \asymp K}\left(\frac{N+k}{m_{1} m_{2} m_{3} m_{4}}\right) .
\end{aligned}
$$

Finally we apply Cauchy's inequality once more and put

$$
h=m_{1} m_{2} m_{3} m_{4}
$$

getting

$$
\begin{aligned}
S_{K M}^{8} & \ll\|\alpha\|^{8}\|\beta\|^{8} K^{2} M^{\varepsilon} \sum_{h \asymp M^{4}}\left|\sum_{k \asymp K}\left(\frac{N+k}{h}\right)\right|^{2} \\
& \ll\|\alpha\|^{8}\|\beta\|^{8} K^{2} M^{\varepsilon}\left(K M^{4}+\sum_{\substack{k_{1}, k_{2} \asymp K \\
k_{1} \neq k_{2}}} \sum_{h \asymp M^{4}}\left(\frac{\left(N+k_{1}\right)\left(N+k_{2}\right)}{h}\right)\right) .
\end{aligned}
$$

Notice that $k_{1} \neq k_{2}$ implies $\left(N+k_{1}\right)\left(N+k_{2}\right)$ is not a square because $k_{1}, k_{2}<N^{1 / 2}$. Applying the Burgess bound [Bu] to the innermost character sum we get

$$
S_{K M}^{8} \ll\|\alpha\|^{8}\|\beta\|^{8} K^{2}\left(K M^{4}+K^{2}\left(M^{4}\right)^{1 / 2}\left(N^{2}\right)^{3 / 16}\right)(M N)^{\varepsilon}
$$

and this concludes the proof. 
Proof of Corollary 4.2. By the Polya-Vinogradov inequality we truncate $L\left(1, \chi_{n}\right)$ to get

$$
\sum_{\substack{N<n \leq N+K \\ n \equiv \nu(8)}} L\left(1, \chi_{n}\right)=\sum_{\substack{N<n \leq N+K \\ n \equiv \nu(8)}} \sum_{m<N} \frac{1}{m}\left(\frac{-4 n}{m}\right)+O\left(N^{\varepsilon}\right) .
$$

We shall consider separately the contributions of square and non-square $m$ 's.

The squares contribute

$$
\sum_{\substack{N<n \leq N+K \\ n \equiv \nu(8)}} \sum_{(k, 4 n)=1} \frac{1}{k^{2}}=\zeta(2) \sum_{\substack{N<n \leq N+K \\ n \equiv \nu(8)}} \sum_{k \mid 4 n} \frac{\mu(k)}{k^{2}}=\frac{3 \zeta(2)}{28 \zeta(3)} K+O(1) .
$$

Therefore

$$
\sum_{\substack{N<n \leq N+K \\ n \equiv \nu(8)}} L\left(1, \chi_{n}\right)=\frac{3 \zeta(2)}{28 \zeta(3)} K+W+O\left(N^{\varepsilon}\right)
$$

where $W$ denotes the contribution of the non-squares terms, i.e.

$$
W=\sum_{\substack{N<n \leq N+K \\ n \equiv \nu(8)}} \sum_{\substack{m<N \\ m \neq k^{2}}} \frac{1}{m}\left(\frac{-4 n}{m}\right)
$$

Dividing the range of summation of $W$ into dyadic intervals it is enough to estimate sums of the type

$$
W_{K M}=M^{-1} \sum_{k \leq K}^{\prime} \sum_{m \asymp M}^{\prime \prime}\left(\frac{N+k}{m}\right)
$$

for some $M \leq N$ where the outer summation restricts $k$ to a fixed arithmetic progression mod 8 and the inner summation restricts $m$ to non-square odd integers belonging to a fixed arithmetic progression mod 4. Applying Polya-Vinogradov inequality for each character sum in $k$ gives

$$
W_{K M} \ll M^{1 / 2+\varepsilon} .
$$

On the other hand by Lemma 4.1

$$
W_{K M} \ll\left(K^{7 / 8}+K M^{-1 / 4} N^{3 / 64}\right) N^{\varepsilon} .
$$


From both estimates it follows that

$$
W_{K M} \ll K^{7 / 8} N^{\varepsilon}+\min \left\{K M^{-1 / 4} N^{3 / 64}, M^{1 / 2}\right\} N^{\varepsilon} .
$$

Choosing $M=K^{4 / 3} N^{1 / 16}$ the corollary is proved.

\section{Proof of Theorem 1.1.}

First we shall derive (1.4) from Corollary 4.2. By (1.1) and (1.2)

$$
\begin{aligned}
S(R, u) & =\frac{1}{\pi} \sum_{R<\sqrt{n} \leq R+u} \sqrt{n} \sum_{d^{2} \mid n} \frac{c_{n / d^{2}}}{d} L\left(1, \chi_{n / d^{2}}\right)+O(1) \\
& =\frac{1}{\pi} \sum_{d \leq R+u} \sum_{R / d<\sqrt{n} \leq(R+u) / d} c_{n} \sqrt{n} L\left(1, \chi_{n}\right)+O(1)
\end{aligned}
$$

where $O(1)$ is introduced to correct the contribution of terms with $d^{2}=$ $n$. This expression and summation by parts in Corollary 4.2 (or the trivial estimate if it does not apply) prove (1.4).

Now we proceed to prove (1.3). We shall not deal with $S(R)$ directly but first smooth by means of the following function

$$
f(x)=\left\{\begin{array}{cl}
x, & \text { if } x \in[0, R], \\
R(R+H-x) / H, & \text { if } x \in[R, R+H], \\
0, & \text { otherwise, }
\end{array}\right.
$$

more precisely we consider

$$
S_{f}(R)=\sum_{n} l(n) f(\sqrt{n})
$$

This sum exceeds $S(R)$ by

$$
S_{f}(R, H)=\sum_{R<\sqrt{n} \leq R+H} l(n) f(\sqrt{n}),
$$

so we have

$$
S(R)=S_{f}(R)-S_{f}(R, H)
$$


The smoothed sum $S_{f}(R)$ will be treated by exponential sums from Section 3 and the short sum $S_{f}(R, H)$ will be treated by character sums from Section 4 .

By Lemma 2.1 we have

$$
S_{f}(R)=\frac{4 \pi}{3} R^{3}+2 \pi H R^{2}+\frac{2 \pi}{3} H^{2} R-1+S_{\widetilde{f}}(R)
$$

where

$$
\widetilde{f}(\xi)=\frac{\sin (2 \pi R \xi)}{2 \pi^{2} \xi^{2}}-\frac{R}{H} \frac{\sin (\pi H \xi)}{\pi^{2} \xi^{2}} \cos (\pi(2 R+H) \xi) .
$$

Note that the application of Lemma 2.1 is justified, although the regularity conditions are not fulfilled, because the involved series converges uniformly on compacta by virtue of Lemma 3.1 .

The first part of $\widetilde{f}$, namely $\sin (2 \pi R \xi) / 2 \pi^{2} \xi^{2}$, contributes to $S_{\widetilde{f}}(R)$ at most $O\left(R^{\varepsilon}\right)$ by estimating the tail of the series using Lemma 3.1. Hence we have

$$
S_{\widetilde{f}}(R)=-\frac{R}{\pi^{2} H} \sum_{n=1}^{\infty} \frac{l(n)}{n} \sin (\pi H \sqrt{n}) \cos (\pi(2 R+H) \sqrt{n})+O\left(R^{\varepsilon}\right) .
$$

Dividing into dyadic intervals by partial summation we get

$$
S_{\widetilde{f}}(R) \ll R H^{-1}\left(H^{1-\varepsilon} N_{1}^{-1}\left|V_{N_{1}}\left(R_{1}\right)\right|+N_{2}^{-3 / 2+\varepsilon}\left|V_{N_{2}}\left(R_{2}\right)\right|\right)+R^{\varepsilon}
$$

for some $N_{1} \leq H^{-2} \leq N_{2}$ and $R_{1}, R_{2}=R+O(H)$. For simplicity we assume $R \leq H^{-2}$. Now we estimate $V_{N_{1}}\left(R_{1}\right)$ and $V_{N_{2}}\left(R_{2}\right)$ by Lemma 3.1, if $N_{1} \leq R$ we get

$$
N_{1}^{-1} V_{N_{1}}\left(R_{1}\right) \ll R^{5 / 16+\varepsilon},
$$

if $R<N_{1} \leq H^{-2}$ we get

$$
N_{1}^{-1} V_{N_{1}}\left(R_{1}\right) \ll\left(H^{-1 / 2}+R^{5 / 24} H^{-5 / 24}\right) H^{-\varepsilon}
$$

and if $N_{2} \geq H^{-2}$ we get

$$
N_{2}^{-3 / 2+\varepsilon} V_{N_{2}}\left(R_{2}\right) \ll\left(H^{1 / 2}+R^{3 / 8} H^{9 / 8}\right) H^{-\varepsilon} .
$$


Collecting the above estimates and substituting in (5.2) we obtain

$$
\begin{aligned}
S_{f}(R)= & \frac{4 \pi}{3} R^{3}+2 \pi H R^{2} \\
& +O\left(\left(R H^{-1 / 2}+R^{9 / 8} H^{-1 / 8}+R^{21 / 16}\right) H^{-\varepsilon}\right) .
\end{aligned}
$$

Now we shall deal with $S_{f}(R, H)$. By Abel's Lemma and (1.4) we have

$$
\begin{aligned}
S_{f}(R, H) & =\frac{R(R+H)}{H} \int_{0}^{H} \frac{S(R, u)}{(R+u)^{2}} d u \\
& =2 \pi H R^{2}+O\left(\left(H^{7 / 8} R^{15 / 8}+H^{2 / 3} R^{83 / 48}+R\right) R^{\varepsilon}\right) .
\end{aligned}
$$

Finally substituting (5.4) and (5.3) in (5.1) and choosing $H=$ $R^{-7 / 11},(1.3)$ follows.

Acknowledgements. We worked on this project at the Universidad Autónoma de Madrid in June 1993. We thank Professor A. Córdoba for making our meeting possible and for encouragement. The second author is grateful for the hospitality and support. This work was finished during our visits to the University of Toronto, we are both grateful for this opportunity. For the first author, this visit was financed by a NATO Collaborative Research Grant (CRG921184) and a CICYT grant, and for the second author by NSERC Grant A5123.

\section{References.}

[Bu] Burgess, D., On character sums and L-series, II. Proc. London Math. Soc. 13 (1963), 524-536. (See also Burgess, D., The character sum estimate with $r=3$. J. London Math. Soc. 33 (1986), 219-226).

[Ch] Chen, Jing-Run. Improvement on the asymptotic formulas for the number of lattice points in a region of the three dimensions (II). Sci. Sinica. 12 (1963), 751-764.

[Fo] Fomenko, O. M., On the problem of Gauss. Acta Arithmetica 6 (1961), 277-284.

[Fr] Fricker, F., Einführung die Gitterpunktlehre. Birkhauser Verlag, 1982.

[Ga] Gauss, C. F., Disquisitiones Arithmeticae. Leipzig, 1801. (English Translation by A. A. Clarke., Yale University Press, 1966). 
[Gr] Grosswald, E., Representation of Integers as sums of squares. Springer Verlag, 1985.

[Gr-Ko] Graham, S. W. and Kolesnik, G., Van der Corput's Method of Exponential Sums. London Math. Soc. Lecture Notes Series 126, 1991.

[Hu] Huxley, M. N., Exponential sums and lattice points II. Proc. London Math. Soc. 66 (1993), 279-301.

[La] Landau, E., Über die Anzahl der Gitterpunkte in gewissen Bereichen. Göttinger Nachr. (1912), 687-771.

[Ti] Titchmarsh, E. C., The Theory of the Riemann Zeta-function. Oxford Clarendon Press, 1951.

[Vi] Vinogradov, I. M., On the number of integer points in a sphere (Russian). Izv. Akad. Nauk SSSR Ser. Mat. 27 (1963), 957-968.

[Wa1] Walfisz, A., Über zwei Gitterpunktprobleme. Math. Ann. 95 (1925), 69-83.

[Wa2] Walfisz, A., Teilerprobleme. Math. Z. 26 (1927), 66-88.

Recibido: 9 de diciembre de 1.993

Revisado: 22 de noviembre de 1.994

Fernando Chamizo*

Departamento de Matemáticas

Universidad Autónoma de Madrid

28049 Madrid, SPAIN

fchamizo@ccuam3.sdi.uam.es

Henryk Iwaniec ${ }^{\dagger}$ Department of Mathematics

Rutgers University

New Brunswick, NJ 08903, U.S.A. iwaniec@math .rutgers . edu

\footnotetext{
* Partially supported by a Fellowship from Caja de Madrid.

$\dagger$ Partially supported by the NSF Grant DMS-9202022 and the NSERC Grant A5123.
} 